\title{
Silencing of the hTERT gene through RNA interference induces apoptosis via Bax/Bcl-2 in human glioma cells
}

\author{
TUO WANG $^{1}$, YAN XUE $^{1}$, MAODE WANG $^{1}$ and QIANG SUN ${ }^{2}$ \\ ${ }^{1}$ Department of Neurosurgery, The First Affiliated Hospital, Xi'an Jiaotong University, Xi'an 710061; \\ ${ }^{2}$ Department of Pharmacology, School of Medicine, Xi'an Jiaotong University, Xi'an 710061, P.R. China
}

Received April 17, 2012; Accepted June 21, 2012

DOI: $10.3892 / o r .2012 .1952$

\begin{abstract}
Glioma cells are characterized by their invasiveness and resistance to conventional therapeutics. The downregulation of human telomerase reverse transcriptase (hTERT) can lead to decreased cell proliferation and/or the induction of apoptotic cell death in cancer cells but has rarely been reported in glioma cells. Here, we assessed the effect of the silencing of the hTERT gene on cell apoptosis and its possible molecular mechanism in T98G glioma cells. We found that the silencing of the hTERT gene in T98G cells significantly decreased cell proliferation and telomerase activity, increased the number of cells in G1 phase and decreased the number of cells in S phase, and induced apoptosis via decreasing the protein level of Bcl-2 and c-Myc and increasing the protein levels of Bax and p53.
\end{abstract}

\section{Introduction}

Gliomas, which originate from the predominant glial tissue in the central nervous system, are the most common types of malignant brain tumors in adults. Patients suffering from malignant gliomas have a life-span between 9 and 12 months after the diagnosis of grade IV and 2 years after the diagnosis of grade III gliomas (1-4). More than $90 \%$ of cancers show activated telomerase, including gliomas. Telomerase activity permits cancer cell immortalization and promotes tumorigenesis. The expression of telomerase and its genetic variation has been correlated with malignant glioma progression (5) and is therefore an important enzyme to target for improving the prognosis and treatment of gliomas (6). Three components of human telomerase have been identified: the RNA component (hTER) (7), the telomerase-associated protein (TEP1) $(8,9)$, and the telomerase reverse transcriptase (hTERT) $(6,10,11)$. Although both hTER and hTERT are necessary for telomerase activity, hTERT is the major determinant of telomerase activity.

Correspondence to: Dr Qiang Sun, Department of Pharmacology, School of Medicine, Xi'an Jiaotong University, Xi'an 710061, P.R. China

E-mail: sunqiang@mail.xjtu.edu.cn

Key words: telomerase, hTERT, glioma, apoptosis
hTERT, the catalytic subunit of telomerase, is the rate-limiting step in the activation of telomerase and is correlated with the pathological grade and type of human glioma.

Apoptosis plays a key role in the pathogenesis of cancers, and the genes relating to this process are of interest in studies on cancer onset and progression. Glioblastomas pose a challenge in neuro-oncology because of their resistance to apoptosis and conventional therapies $(12,13)$. Bax and Bcl-2 are transcriptional targets of the tumor suppression protein p53, which is responsible for the induction of cell cycle arrest and/or apoptosis in response to DNA damage. The progression of cancer mainly depends on the balance between pro-apoptotic proteins, such as Bax, and anti-apoptotic proteins, such as Bcl-2 (14). The inhibition of hTERT rapidly induces apoptosis in gastric cancer, but this effect has rarely been reported in gliomas.

In this study, we used siRNA to downregulate hTERT in T98G cells and investigated the effect of hTERT on T98G cell proliferation, apoptosis and cell cycle progression. We also explored its possible molecular mechanism.

\section{Materials and methods}

Cell culture. The human glioblastoma cell line T98G was procured from the Huaxi Medical Center (Sichuan University, Chengdu, Sichuan, China). T98G was derived from a human glioblastoma multiforme tumor. We propagated T98G cells in DMEM (Gibco-BRL, Carlsbad, CA, USA) supplemented with $10 \%$ fetal bovine serum (Invitrogen, Carlsbad, CA, USA) and antibiotics in a humidified incubator containing $5 \% \mathrm{CO}_{2}$ at $37^{\circ} \mathrm{C}$.

Small-interfering RNA design and transfection. The cDNA sequence of the human telomerase reverse transcriptase gene (hTERT) (GenBank accession number NM_198253) was used to design small-interfering RNA (siRNA). The specificity of the siRNA sequence was confirmed through BLAST searches, and the sequence did not show any homology to other known human genes. A random coding sequence of siRNA was used as a negative control. The specific small-interfering RNAs (siRNAs) were synthetized and sequenced by Shanghai GenePharma Co., Ltd. (Shanghai, China). For hTERT, the siRNA sense sequence was 5'-CGGUGUACGCCGAGACCA ATT-3', and the anti-sense sequence was 5'-UUGGUCUCGG 
CGUACACCGGG-3'. The negative-control siRNA sense sequence was 5'-UUCUCCGAACGUGUCACGUTT-3', and the anti-sense sequence was 5'-ACGUGACACGUUCGGA GAATT-3'. The most effective construct was selected based on the percentage knockdown of hTERT at both the mRNA and protein levels. For transfection, $2 \times 10^{5}$ cells were seeded into each well of a 6-well tissue culture plate (Costar). The next day (when the cells were 70-80\% confluent), the culture medium was aspirated, and the cell monolayer was washed with prewarmed sterile phosphate-buffered saline (PBS). The cells were transfected with Lipofectamine 2000 reagent (Invitrogen) in accordance with the manufacturer's protocol. The cells were continuously cultured until they were harvested for analysis. The transfection efficiency was monitored with the expression of carboxyfluorescein (FAM) under a phase-contrast fluorescent microscope (Olympus IX71, Japan).

Reverse transcription-polymerase chain reaction (RT-PCR) and western blotting to examine the hTERT $m R N A$ and protein levels. RT-PCR and western blotting were performed to examine the downregulation of hTERT mRNA and protein levels, respectively, after the knockdown of hTERT. The following primer sequences were used for the PCR amplification of hTERT: sense strand, 5'-ATGGCTGCGTGGTGAAC TTG-3', and antisense strand, 5'-AGGTGAGACTGGCTCT GATGG-3'. The primers were used to amplify $1,000 \mathrm{ng}$ of total RNA with a single-step RT-PCR kit (Invitrogen) with a PCR cycler (Eppendorf, Westbury, NY, USA) at an annealing temperature of $56^{\circ} \mathrm{C}$. Western blotting was performed with an hTERT antibody (Santa Cruz Biotechnology, Santa Cruz, CA, USA) to determine hTERT protein levels as described below. Both RT-PCR and western blotting images were quantified using Gel-Pro Analyzer software (Media Cybernetics, Silver Spring, MD, USA).

Flow cytometry cell cycle analysis. T98G cells $\left(1 \times 10^{7}\right)$ were cultured in each well of a 6-well plate to 70-80\% confluence with normal culture medium. The cell were treated with hTERT siRNA (100 nM) for 2 or 3 days, trypsinized, and stained with propidium iodide with the Cellular DNA Flow Cytometric Analysis Reagent Set (Boehringer Mannheim, Indianapolis, IN, USA). The cells were harvested and fixed with $3 \mathrm{ml}$ of ice-cold $70 \%$ ethanol overnight. Then, the cells were incubated with RNase A $(1 \mathrm{mg} / \mathrm{ml}$; Sigma, St. Louis, MO, USA) for $10 \mathrm{~min}$ at room temperature. The DNA was stained with propidium iodide $(50 \mu \mathrm{g} / \mathrm{ml})$ for at least $1 \mathrm{~h}$ at $4^{\circ} \mathrm{C}$, and the DNA content was determined with flow cytometry (Beckman Coulter, San Diego, CA, USA). The data were analyzed with CellQuest software (Becton-Dickinson, San Jose, CA, USA).

Telomerase activity (TRAP) assay. Telomerase activity was determined with a PCR-based telomeric repeat amplification protocol (TRAP) enzyme-linked immunosorbent assay (ELISA) kit (Roche, Mannheim, Germany) according to the manufacturer's protocol. In brief, T98G cells were collected $48 \mathrm{~h}$ after siRNA transfection. The cells were washed three times with cold PBS, homogenized in $200 \mu$ l cell lysis buffer, and incubated on ice for $30 \mathrm{~min}$. For the TRAP reaction, $2 \mu \mathrm{l}$ of cell extract was added to $25 \mu 1$ of reaction mixture, and sterile water was added to a final volume of $50 \mu 1$. PCR was then performed as follows: primer elongation $\left(20 \mathrm{~min}, 25^{\circ} \mathrm{C}\right)$, telomerase inactivation $\left(5 \mathrm{~min}, 94^{\circ} \mathrm{C}\right)$, product amplification for 30 cycles $\left(94^{\circ} \mathrm{C}\right.$ for $30 \mathrm{sec}, 50^{\circ} \mathrm{C}$ for $30 \mathrm{sec}$, and $72^{\circ} \mathrm{C}$ for $90 \mathrm{sec})$ and then balance $\left(10 \mathrm{~min}\right.$ at $\left.72^{\circ} \mathrm{C}\right)$. A total of $5 \mu 1$ of PCR products was added to a streptavidin-coated 96-well plate and hybridized to a digoxigenin (DIG)-labeled telomeric repeat-specific detection probe. The immobilized PCR products were detected with peroxidise-conjugated anti-DIG antibody. After addition of the stop reagent, the plate was assessed with a plate reader at a wavelength of $450 \mathrm{~nm}$ within $30 \mathrm{~min}$.

MTT assay. T98G cells were incubated in 96-well plates, with each well containing $200 \mu \mathrm{l}$ of medium. The cells were divided into the three following groups: i) blank group, ii) control siRNA group, and ii) hTERT siRNA group. The transfection of siRNAs was performed the following day, as previously described (15). The rate of cellular proliferation was measured every $24 \mathrm{~h}$ for $120 \mathrm{~h}$. At the end of each time point, $20 \mu 1$ of $5 \mathrm{mg} / \mathrm{ml}$ MTT (Sigma) was added to each well. Four hours later, $200 \mu \mathrm{l}$ of DMSO was added to the MTT-treated wells, and the absorption at $492 \mathrm{~nm}$ was determined with a spectrometer. Each experimental condition was performed in triplicate.

Apoptosis assay. Flow cytometry assays and TUNEL assays were performed to detect cell apoptosis. A total of $1 \times 10^{6}$ cells were transfected with siRNA. At $30 \mathrm{~h}$ post-transfection, the cells were harvested, washed twice with PBS, and resuspended in $200 \mu \mathrm{l}$ Annexin $\mathrm{V}$ binding buffer (10 mM HEPES, $140 \mathrm{mM}$ $\mathrm{NaCl}, 2 \mathrm{mM} \mathrm{MgCl} 2,5 \mathrm{mM} \mathrm{KCl}$, and $2.5 \mathrm{mM} \mathrm{CaCl}_{2}, \mathrm{pH}$ 7.4). A total of $10 \mu 1$ FITC-conjugated Annexin V (Beijing Biosea Biotechnology Co., Beijing, China) was added according to the manufacturer's protocol. After incubation for $20 \mathrm{~min}$ at room temperature in the dark, another $400 \mu 1$ of binding buffer was added, and the samples were immediately analyzed using FACSCalibur. In total, $1 \times 10^{4}$ cells were collected and analyzed with CellQuest software. Apoptotic cells are expressed as a percentage of total cells.

The TUNEL assay was performed to detect apoptosis as a marker of cell death. Briefly, T98G cells were fixed with $4 \%$ paraformaldehyde for $10 \mathrm{~min}$ and then incubated for $60 \mathrm{~min}$ with TdT. Streptavidin-HRP was added to the samples, which were then incubated in the dark for $30 \mathrm{~min}$ and incubated for 10 min with DAB. Positively stained cells were visualized and photographed using a microscope.

Western blotting for molecules involved in cell apoptosis regulation in vitro. Cells were lysed in M-PER Mammalian Protein Extraction Reagent (Pierce Biotechnology, Inc., Rockford, IL, USA) supplemented with a protease inhibitor cocktail (Roche, Indianapolis, IN, USA) followed by centrifugation at 12,000 rpm for $10 \mathrm{~min}$. After centrifugation, the cell lysates were collected, and the protein concentrations of the cell lysates were measured. Proteins (10-20 $\mu \mathrm{g})$ were resolved through SDS-PAGE and then transferred to PVDF membranes (Bio-Rad Laboratories, Hercules, CA, USA). The blots were incubated with primary antibodies in $3 \% \mathrm{BSA} / \mathrm{TBST}$ at $4^{\circ} \mathrm{C}$ overnight followed by incubation with secondary antibodies 

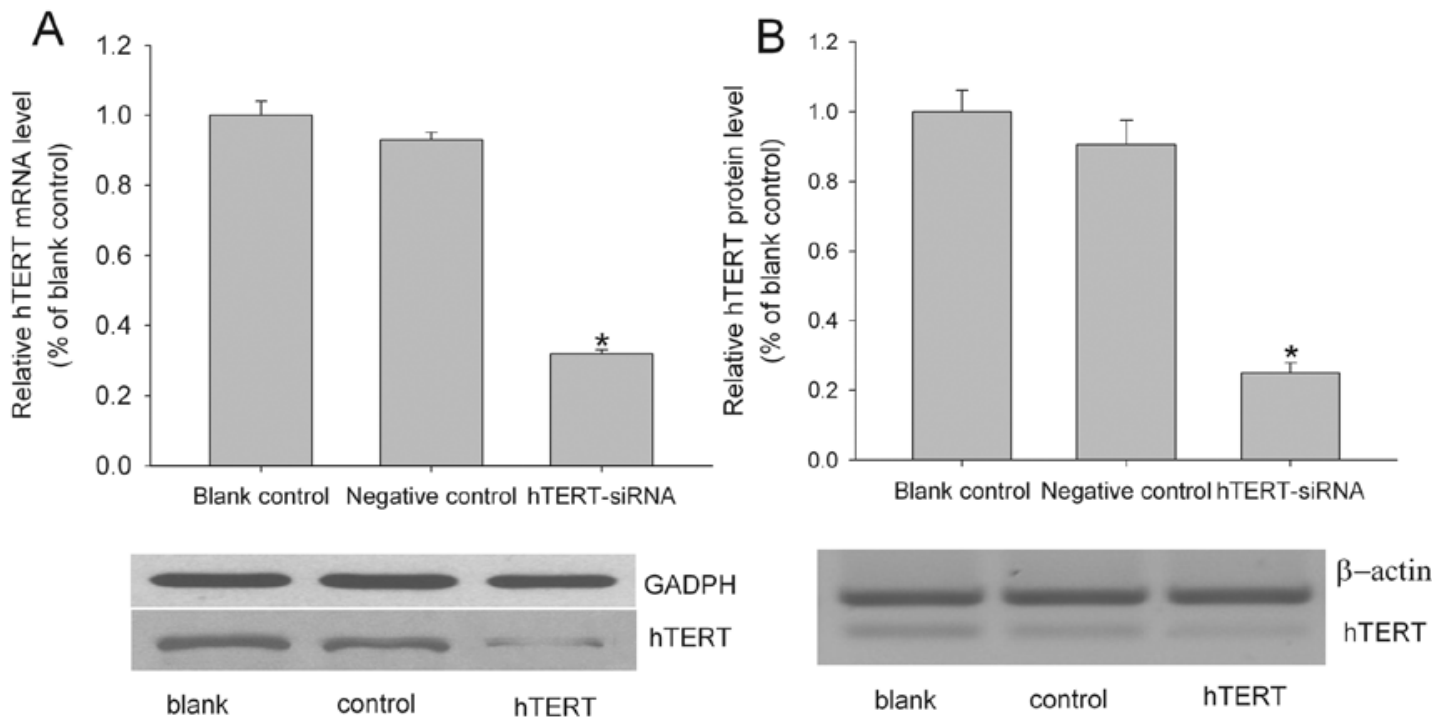

Figure 1. The transfection of hTERT siRNA inhibits hTERT expression at both the mRNA and protein level. (A) RT-PCR results for hTERT mRNA expression. The values indicate the mean $\pm \mathrm{SD}$ of 3 independent experiments in each group (" $\mathrm{P}<0.01$ compared to the control mean values). (B) Western blotting of hTERT protein levels. The data are representative of 3 independent experiments (" $\mathrm{P}<0.01$ compared to the control mean values).

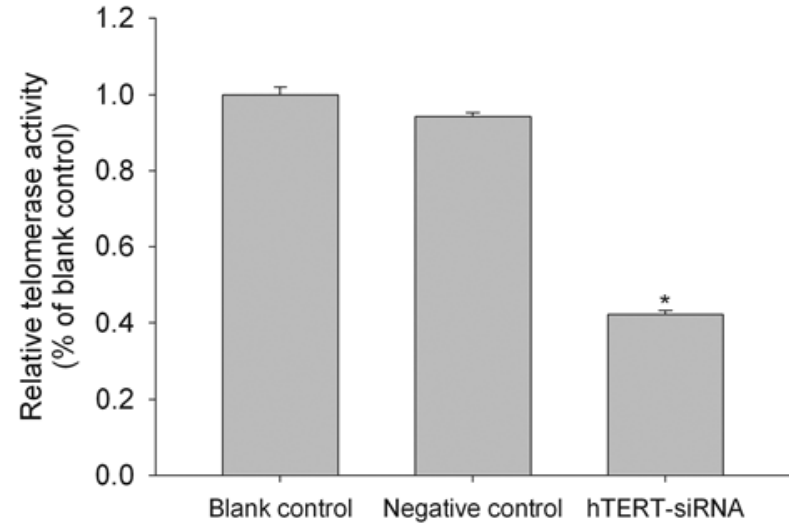

Figure 2. Telomerase activity levels after transfection with hTERT siRNA. Telomerase activity levels quantified with TRAP assays in T98G cells at $48 \mathrm{~h}$ after a 6-h exposure to hTERT siRNA or control siRNA and in untransfected cells. The data are shown as the mean \pm SD (error bar) of 3 experiments $($ $\mathrm{P}<0.01$ compared to the control mean values).

at room temperature for $1 \mathrm{~h}$. The protein signals were detected with the ECL method (16).

Statistical analysis. The mean and standard deviation (SD) were calculated for all of the quantitative data. The results were statistically evaluated using a one-way analysis of variance (ANOVA). The least significant difference method was used to compare the mean values of control or negative control siRNA-treated groups with hTERT siRNA-treated groups. A value of $\mathrm{P}<0.05$ was considered statistically significant.

\section{Results}

Downregulation of hTERT $m R N A$ and protein levels in T98G cells. We initially tested three sets of siRNAs for hTERT knockdown. The preliminary results showed one set being particular effective (data not shown); thus, all of the experi- ments described in this report were performed with this siRNA (for the sequence, see the methods section).

The hTERT siRNA was transiently transfected into the T98G glioma cell line. After $48 \mathrm{~h}$, the hTERT mRNA and protein levels were quantified with real-time RT-PCR and western blotting, respectively. As shown in Fig. 1A, hTERT siRNA transfection significantly reduced the amount of hTERT mRNA. The level of hTERT mRNA in the hTERT siRNA-treated group was $\sim 40 \%$ of the blank group. The control siRNA had no effect on the hTERT mRNA level. The hTERT siRNA was also successful in knocking down hTERT protein expression. As shown in Fig. 1B, although the $\beta$-actin internal control showed equal loading among the three groups, the level of hTERT protein was noticeably lower in the hTERT siRNA-treated group compared to both the blank and the negative siRNA-treated groups, suggesting that the hTERT siRNA treatment could effectively reduce the hTERT protein level.

Downregulation of telomerase activity in brain glioma cells after hTERT siRNA transfection. The level of telomerase activity in human gliomas has been shown to correlate significantly with hTERT mRNA expression (17). Consistent with this result, we found that the hTERT siRNA-transfected cells showed a $42 \%$ reduction in telomerase activity, as determined with a PCR-based telomeric repeat amplification protocol (TRAP) ELISA (Fig. 2).

hTERT siRNA inhibited cell viability in vitro. Decreased telomerase activity is associated with arrested cell growth; therefore, we determined whether the hTERT siRNA-induced reduction in telomerase activity affected the cell viability of the T98G cell line. The cells were transfected with hTERT siRNA, and the number of viable cells was determined with the MTT assay every $24 \mathrm{~h}$ for 4 days. As shown in Fig. 3, hTERT siRNA significantly decreased the percentage of viable cells in the T98G cell line. The decrease was rapid: only $\sim 57.7 \%$ 


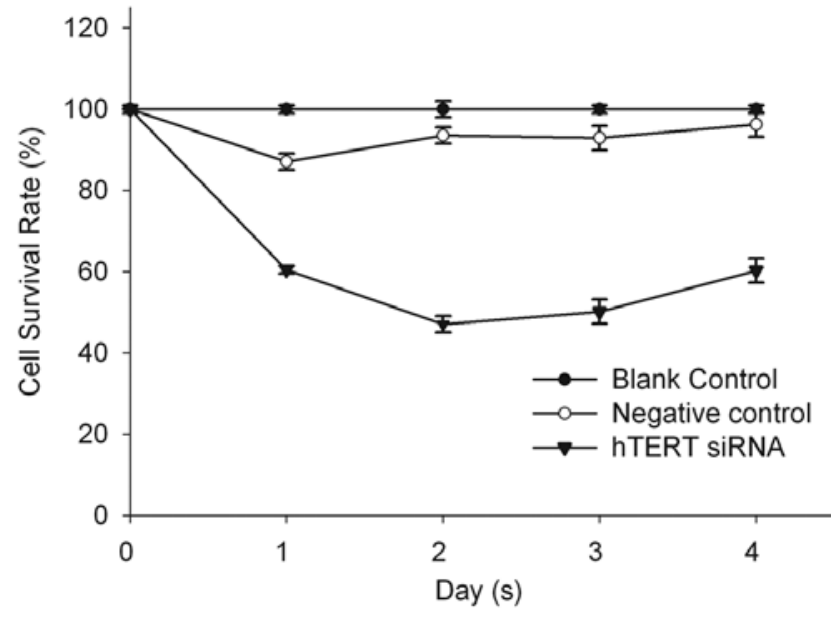

Figure 3. The determination of the cell viability of T98G cells after transfection with hTERT siRNA. MTT assay for the determination of cell viability. The data are the mean $\pm \mathrm{SD}$ of 6 independent experiments.

of cells were viable after $24 \mathrm{~h}$, and only $47 \%$ of cells survived after $48 \mathrm{~h}$. However, the inhibitory effect of the siRNA was temporary, and the T98G cells began proliferating 3 days after the exposure to siRNA.

The effect of hTERT siRNA on the cell cycle. To determine whether hTERT siRNA affected the cell cycle of malignant glioma cells, flow cytometry was performed. As shown in Fig. 4, the flow cytometry assay showed the after transfection with hTERT siRNA, the number of cells in G1 phase was increased, but the number of cells in $\mathrm{S}$ phase was decreased. There was no alteration in the cell population in subG1, $\mathrm{S}$, or G2/M phase in the negative-control group.

The decrease in cell viability caused by hTERT siRNA is due to an increase in apoptosis. To determine whether the decrease in cell viability caused by the hTERT siRNA was due to an increase in apoptosis, we determined the number of early apoptotic cells in the untransfected, negative controland hTERT siRNA-transfected cells with Annexin V-FITC and propidium iodide (PI) labeling followed by fluorescence- activated cell sorting (FACS). As shown in Fig. 5, $48 \mathrm{~h}$ after siRNA transfection, the number of early apoptotic cells was increased significantly. Cell apoptosis was then examined using a TUNEL assay. hTERT siRNA dramatically increased the number of cells that stained positive for TUNEL within the nucleus (Fig. 6), indicating a high incidence of apoptosis.

hTERT siRNA downregulates the molecules involved in apoptosis. To confirm the molecular mechanism of the inhibition of cell apoptosis after the downregulation of hTERT, we determined the protein levels of the important molecules involved in this process (Fig. 7). A western blotting assay showed that the levels of several proteins involved in the apoptotic pathway were different. The expression level of Bcl-2 and c-Myc was decreased whereas the expression level of Bax and p53 was increased after the treatment of T98G cells with hTERT siRNA (Fig. 7).

\section{Discussion}

This study shows that in T98G glioma cells, siRNAs targeting the hTERT gene can be efficiently delivered and results in the rapid inhibition of telomerase activity and cell growth. The inhibition of cell growth is associated with cell cycle arrest and the promotion of cell apoptosis through transcriptional and/or translational upregulation and/or downregulation of the molecules involved in this process.

RNA interference has emerged as an effective method for the specific inhibition of gene expression both in vitro and in vivo. Telomerase plays a key role in cellular immortality and tumorigenesis. Telomerase is a distinctive candidate for the targeted gene therapy of malignant gliomas because the vast majority of malignant gliomas express telomerase activity, whereas normal brain tissues do not (18-20). Telomerase and its major catalytic subunit hTERT are upregulated in most cancers, including glioblastomas $(17,21)$. Moreover, hTERT expression has been correlated with poor survival in glioblastoma patients (22).

Previous studies have demonstrated that the downregulation of hTERT in glioblastoma cells is correlated with a decrease in cell viability, proliferation, tumor cell migration, and invasion through the downregulation of the molecules

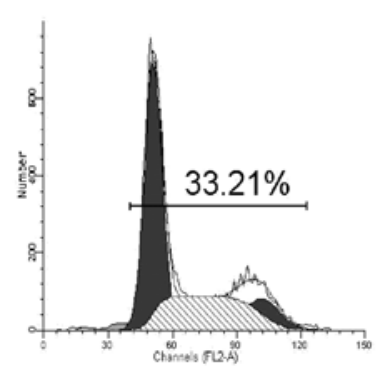

Black control

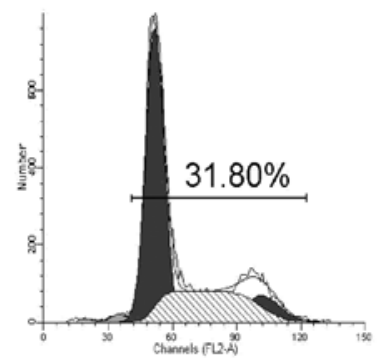

Negative control

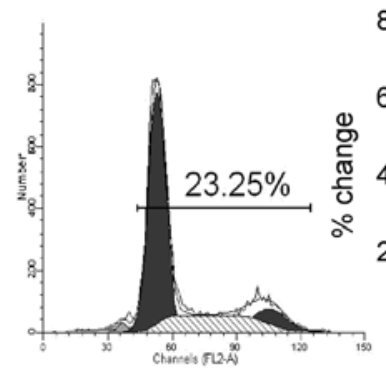

hTERT SIRNA

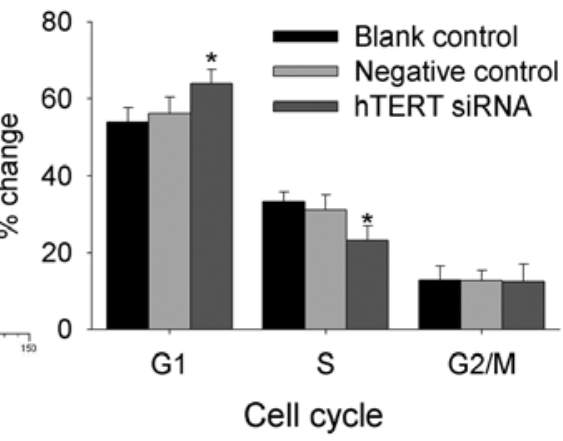

Figure 4. Cell cycle analysis after transfection with hTERT siRNA for $48 \mathrm{~h}$. (A) FACS histograms of T98G cells showing cell cycle arrest. The cells were treated with $50 \mu \mathrm{g} / \mathrm{ml}$ propidium iodide for $20 \mathrm{~min}$ at $4^{\circ} \mathrm{C}$ in the dark prior to cell cycle analysis. An increase in the cell population in $\mathrm{G} 1$ phase indicated cell cycle arrest. (B) Quantitative representation of FACS data of the population of cells in G1, S and G2/M phase. The data are representative of 4 independent experiments $\left({ }^{*} \mathrm{P}<0.01\right.$ compared with the mean values of control siRNA-treated cells). 


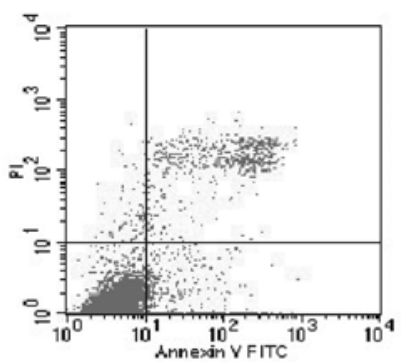

Blank control

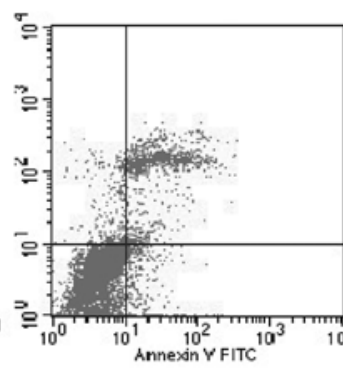

Negative control

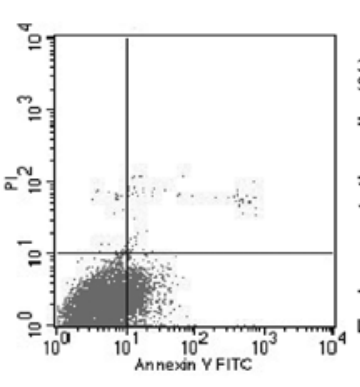

hTERT siRNA

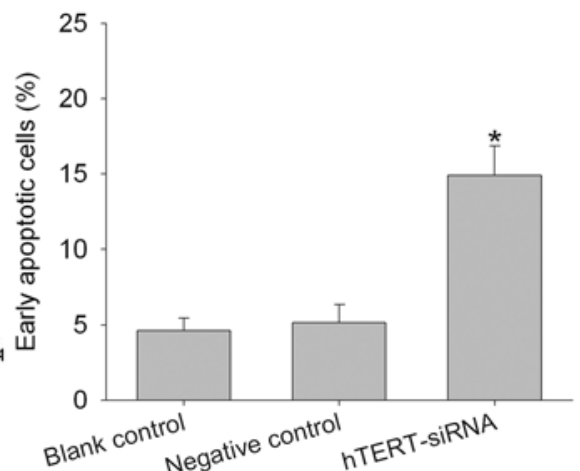

Figure 5. Flow cytometry analysis of early cell apoptosis after hTERT siRNA. T98G cells were transfected with hTERT siRNA. At 48 h post-transfection, the percentage of cell apoptosis was analyzed with flow cytometry with Annexin V-FITC. The M1 numbers indicate the corresponding proportions of apoptotic cells that display positive Annexin V-FITC labeling. The data indicate that hTERT siRNA significantly promotes T98G cell early apoptosis. Columns representing the flow cytometry data are presented on the right. The data are means $\pm \mathrm{SD}$ of values from three independent experiments. ${ }^{*}<0.05$ compared with the mean values of control siRNA-treated cells.

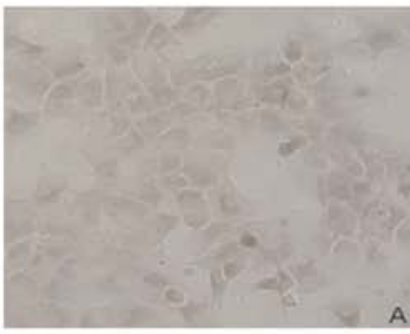

Blank control

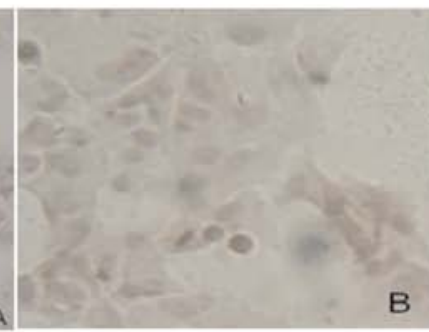

Negative control

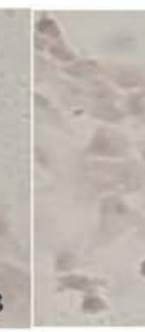

(

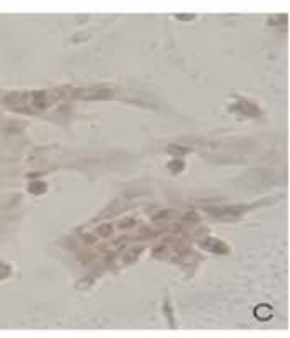

hTERT SiRNA

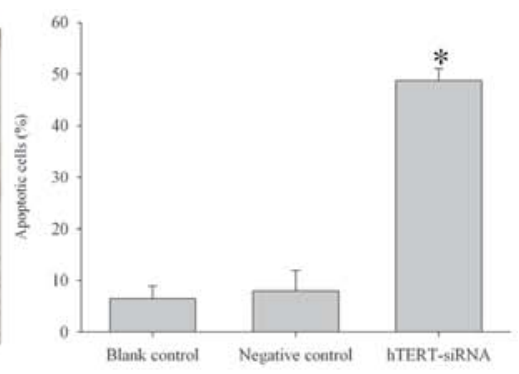

Blank control Negative control bTERT-siRNA

Figure 6. Effect of hTERT-siRNA on the apoptosis of brain cancer T98G cells. The apoptosis of brain cancer cells induced by hTERT siRNA. Apoptotic cells were detected with a TUNEL assay. The columns representing the TUNEL data are presented at left. The data shown indicate that hTERT siRNA significantly promotes T98G cell apoptosis. The experiments were performed in triplicate, with similar results (" $\mathrm{P}<0.01$ compared with the mean values of control siRNAtreated cells).
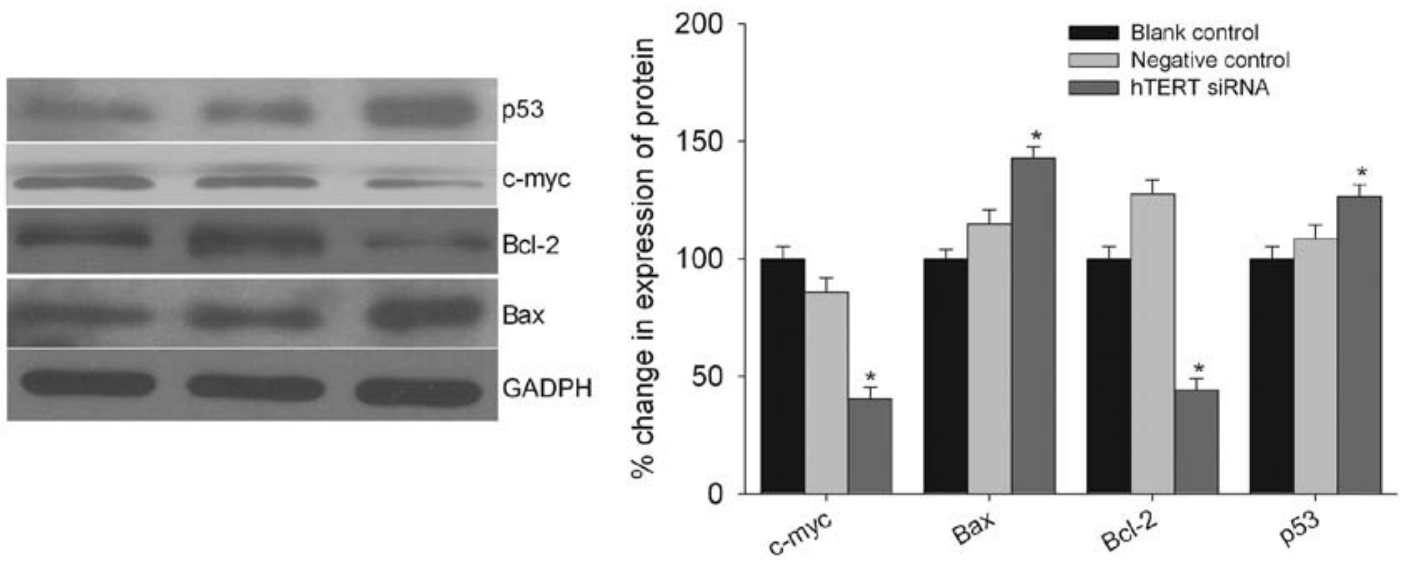

Figure 7. Effect of hTERT-siRNA on some molecules involve in cell apoptosis. (A) Western blotting of p53, c-Myc, Bcl-2 and Bax protein level. The GAPDH was used as an internal control. (B) Columns representing the western blotting data are presented on the right. The data are the mean \pm SD of 6 samples. ( $\mathrm{P}<0.01$ compared with the mean values of control siRNA-treated samples).

involved in these processes and cell cycle inhibition (17,21). In the present study, siRNA directed against hTERT resulted in $>70 \%$ suppression of hTERT at the mRNA and protein levels. Furthermore, siRNA targeting hTERT significantly inhibited cell proliferation and increased apoptosis by downregulating hTERT expression and decreasing telomerase activity in T98G human glioma cells.
In cancer cells, the stabilization of telomeres through the reactivation of telomerase has been suggested to be a crucial step during cellular immortalization and tumorigenesis. Moreover, telomerase inhibition is associated with the induction of apoptosis and senescence. Earlier studies have shown that the selective silencing of hTERT using hTERT siRNA and oligonucleotides targeting the RNA component of telomerase 
induces both apoptosis and senescence in Barrett's adenocarcinoma cells $(5,18)$. In our present study, silencing hTERT using hTERT siRNA induced apoptosis in T98G glioma cells.

c-Myc contributes to apoptosis via its interaction with a number of apoptotic pathways. Pathways involving p53 and Bax (Bcl-2-associated $\mathrm{X}$ protein) have been shown to be activated by c-Myc (6). In addition, Bcl-2 suppresses c-Myc-induced apoptosis without affecting the ability of c-Myc to regulate the progression of the cell cycle from G1 phase to $\mathrm{S}$ phase. c-Myc-induced tumorigenesis is the result of the suppression of apoptosis by cooperating oncogenes and the activation of $\mathrm{S}$ phase by c-Myc, leading to cell proliferation $(23,24)$. siRNAmediated c-Myc downregulation resulted in an inhibition of cellular proliferation and clonogenic growth, the inhibition of G1-S phase cell cycle progression, and a decrease in human telomerase reverse transcriptase (hTERT) expression and telomerase activity in human medulloblastoma cells (25).

Anti-apoptotic Bcl-2 family members are highly overexpressed in malignant gliomas. The induction of apoptosis by downregulating hTERT expression and decreasing telomerase activity was shown in changes in the expression levels of proteins responsible for the regulation of apoptosis. Bax and Bcl-2 are the two principal genes involved in the regulation of apoptosis. Previous studies have demonstrated that during apoptosis induction, bax protein levels are upregulated, which has a well-known pro-apoptotic effect, $\mathrm{Bcl}-2$, which protects cells from apoptosis, is downregulated. According to our results, the anticancer cell growth inhibition is due to the deregulation of apoptosis induction.

The p53 tumor suppressor is another cell cycle regulator that is frequently altered in brain tumors. During cell DNA damage or cytotoxic stress, there is an increase in p53 protein levels that induces cell growth arrest, DNA repair mechanisms, and apoptosis (26-28).

In conclusion, our study demonstrated that the knockdown of hTERT effectively inhibited the cell viability of human glioblastoma cells by increasing the positive index of apoptotic cells via decreasing the expression of Bcl-2 and c-Myc and cell cycle arrest at G0/G1 phase. Therefore, hTERT siRNA offers a potential therapeutic regimen for effectively controlling the growth of human glioblastoma cells.

\section{Acknowledgements}

This study was supported in part by the Shaanxi Provincial scientific and technological research projects (no. 2011K12-56).

\section{References}

1. Lino MM and Merlo A: PI3Kinase signaling in glioblastoma. J Neurooncol 103: 417-427, 2011.

2. Zhao P, Wang C, Fu Z, et al: Lentiviral vector mediated siRNA knock-down of hTERT results in diminished capacity in invasiveness and in vivo growth of human glioma cells in a telomere length-independent manner. Int J Oncol 31: 361-368, 2007.

3. Li C, Zhou C, Wang S, et al: Sensitization of glioma cells to tamoxifen-induced apoptosis by Pl3-kinase inhibitor through the GSK-3beta/beta-catenin signaling pathway. PLoS One 6: e27053, 2011.
4. Prados MD and Levin V: Biology and treatment of malignant glioma. Semin Oncol 27: 1-10,2000.

5. Ponnala S, Chetty C, Veeravalli KK, Dinh DH, Klopfenstein JD and Rao JS: MMP-9 silencing regulates hTERT expression via betal integrin-mediated FAK signaling and induces senescence in glioma xenograft cells. Cell Signal 23: 2065-2075, 2011.

6. Shervington A, Cruickshanks N, Wright H, et al: Glioma: what is the role of c-Myc, hsp90 and telomerase? Mol Cell Biochem 283: 1-9, 2006

7. Feng J, Funk WD, Wang SS, et al: The RNA component of human telomerase. Science 269: 1236-1241, 1995.

8. Harrington L, McPhail T, Mar V, et al: A mammalian telomeraseassociated protein. Science 275: 973-977, 1997.

9. Nakayama JI, Saito M, Nakamura H, Matsuura A and Ishikawa F: TLP1: a gene encoding a protein component of mammalian telomerase is a novel member of WD repeats family. Cell 88: 875-884, 1997.

10. Meyerson M, Counter CM, Eaton EN, et al: hEST2, the putative human telomerase catalytic subunit gene, is up-regulated in tumor cells and during immortalization. Cell 90: 785-795, 1997.

11. Kondo S, Kanzawa T, Germano IM, Kondo Y, Ito H and Kyo S: Inhibition of telomerase activity in malignant glioma cells correlates with their sensitivity to temozolomide. $\mathrm{Br} \mathrm{J}$ Cancer 89: 922-929, 2003.

12. Ferguson SD: Malignant gliomas: diagnosis and treatment. Dis Mon 57: 558-569, 2011.

13. Furnari FB, Fenton T, Bachoo RM, et al: Malignant astrocytic glioma: genetics, biology, and paths to treatment. Genes Dev 21: 2683-2710, 2007.

14. Adams JM and Cory S: The Bcl-2 apoptotic switch in cancer development and therapy. Oncogene 26: 1324-1337, 2007.

15. Xue Y, Li L, Zhang D, et al: Telomerase suppression initiates PML-dependent p53 activation to inhibit bladder cancer cell growth. Oncol Rep 24: 1551-1559, 2010

16. Zhang Y, Cheng Y, Zhang L, et al: Inhibition of eEF-2 kinase sensitizes human glioma cells to TRAIL and down-regulates Bcl-xL expression. Biochem Biophys Res Commun 414: 129-134, 2011.

17. George J, Banik NL and Ray SK: Knockdown of hTERT and concurrent treatment with interferon-gamma inhibited proliferation and invasion of human glioblastoma cell lines. Int J Biochem Cell Biol 42: 1164-1173, 2010.

18. Shammas MA, Koley H, Batchu RB, et al: Telomerase inhibition by siRNA causes senescence and apoptosis in Barrett's adenocarcinoma cells: mechanism and therapeutic potential. Mol Cancer 4: 24,2005

19. Chen $\mathrm{CH}$ and Chen RJ: Prevalence of telomerase activity in human cancer. J Formos Med Assoc 110: 275-289, 2011.

20. Shay JW and Wright WE: Role of telomeres and telomerase in cancer. Semin Cancer Biol 21: 349-353, 2011.

21. George J, Banik NL and Ray SK: Combination of hTERT knockdown and IFN-gamma treatment inhibited angiogenesis and tumor progression in glioblastoma. Clin Cancer Res 15: 7186-7195, 2009

22. Wang YF, Wang DL, Shi GS and Huang H: Expressions of hTERT, HIF-1alpha and CD105 in gliomas and their clinical significance. Zhonghua Bing Li Xue Za Zhi 35: 681-682, 2006 (In Chinese).

23. Packham G and Cleveland JL: c-Myc and apoptosis. Biochim Biophys Acta 1242: 11-28, 1995.

24. Lutz W, Leon J and Eilers M: Contributions of Myc to tumorigenesis. Biochim Biophys Acta 1602: 61-71, 2002.

25. von Bueren AO, Shalaby T, Oehler-Janne C, et al: RNA interference-mediated c-MYC inhibition prevents cell growth and decreases sensitivity to radio- and chemotherapy in childhood medulloblastoma cells. BMC Cancer 9: 10, 2009.

26. Louis DN: The p53 gene and protein in human brain tumors. J Neuropathol Exp Neurol 53: 11-21, 1994.

27. Levine AJ, Hu W and Feng Z: The P53 pathway: what questions remain to be explored? Cell Death Differ 13: 1027-1036, 2006.

28. Krakstad C and Chekenya M: Survival signalling and apoptosis resistance in glioblastomas: opportunities for targeted therapeutics. Mol Cancer 9: 135, 2010. 